\title{
Genetics tells tall tales
}

Human height has posed an emblematic challenge to geneticists searching for the link between genes and complex traits and diseases. It's strongly heritable - how tall one's parents are is $80-90 \%$ predictive of one's own stature. But studies scanning the genomes of tens of thousands of individuals for gene variants associated with height have come up short: around 50 variants have been identified, but together they account for only $5 \%$ or so of height's heritability.

Many geneticists have begun to refer to this gap - seen in almost all complex traits and diseases investigated - as the 'missing heritability' of the genome $e^{1}$. But a study on the genetics of height published online in Nature Genetics this week ${ }^{2}$ suggests that this heritability may not be missing - it may simply be buried deeper than previously thought, in a multitude of genetic variants that have tiny effects individually.

Genome-wide association studies (GWAS) scan the genomes of thousands of people at a time, looking for common single-letter mutations called SNPs (sin-

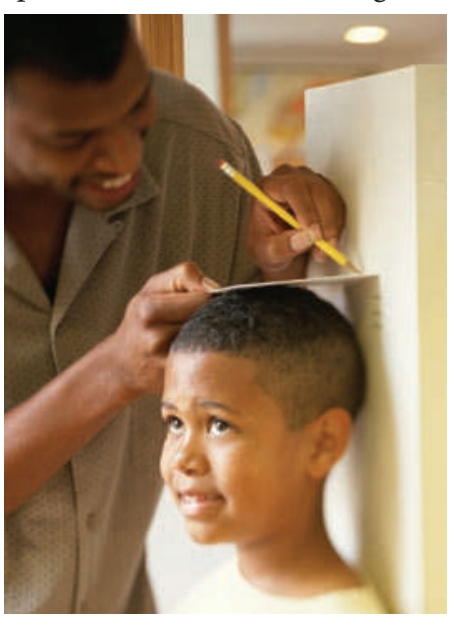

How tall will he grow? individuals. associated with a trait or disease are, on their own, likely to be quite small. Second, in order to spot them researchers will have to study groups of many hundreds of thousands of

The problem of the missing heritability has led some researchers to question the very idea that the common genetic variants GWAS are designed to pick up will explain complex traits and diseases. Instead, they have shifted their focus to search for rare variants - by resequencing genes they suspect are involved, or whole genomes or exomes (the proteincoding sequences) in people with the trait. A study published online in Nature last week $^{3,4}$, for example, identified a handful of rare variants of a specific gene that could raise a person's susceptibility to certain autoimmune diseases.

\section{Rare or common?}

Although rare variants are known to be at play in some complex traits, abandoning GWAS is premature, not to say illogical, says David Altshuler, director of medical and population genetics at the Broad Institute in Cambridge, Massachusetts. "I don't think you could say this new paper resolves the issue," he says. "Studies like this simply remind us that we shouldn't leap to conclusions about what we haven't yet explained."

Increasingly, most researchers agree that there's no either/or answer. "I think the most likely scenario is there's a spectrum of variance," says Visscher. His group's study, though — and a similar analysis published last year on schizophrenia $^{5}$ - suggests that there may be many more meaningful common variants to uncover, although that won't be easy. "If the true state of nature is that there are really very many causal variants, each with a small effect on disease risk or trait," says Visscher, "then that's not the fault of GWAS, that's just the way it is."

\section{Alla Katsnelson}

1. Maher, B. Nature 456, 18-21 (2008).

2. Yang, J. et al. Nature Genet. advance online publication doi:10.1038/ng.608 (2010).

3. Surolia, I. et al. Nature advance online publication doi:10.1038/nature09115 (2010)

4. Katsnelson, A. Nature doi:10.1038/news.2010.300 (2010)

5. International Schizophrenia Consortium Nature 460, 748-752 (2009).
The results suggest two things, says Visscher. First, the effects of many common variants 